\title{
La proporción de las mujeres en la epigrafía funeraria romana del interior de Hispania
}

\author{
Enrigue Gozalbes Cravioto *
}

\section{RESUMEN SUMMARY}

El presente trabajo analiza un aspecto concreto de los epitafios latinos de

Hispania. A partir del mismo se detecta la existencia de proporciones diferentes en la presencia de las mujeres, que aparecen en mayor número en Andalucía y costa mediterránea, $y$ en proporciones mucho más exiguas en regiones del interior. Dicha diferencia deriva de posibles situaciones diferentes en la condición social de la mujer.
The present work studies a concrete aspect of the Latin epitaphs of Hispania. From same is detected the different proportions existence of women, that appear in greater number in the coastal regions, and in proportions much more reduced in the interior. Said diference derives, without doubt, from different situations in the social condition from the woman.

Los epitafios latinos constituyen un documento relativamente numeroso legado por el mundo antiguo. En todas las regiones de las Hispaniae, incluso del conjunto del Imperio romano, aparecen con una notable asiduidad, y vienen siendo objeto de recopilación y de análisis desde el Renacimiento. Basta con revisar el venerable CIL. y sus suplementos, las publicaciones de los nuevos epígrafes aparecidos con posterioridad (por ejemplo, salvando la dispersión, en “L'Année Épigraphique», o en la

* Universidad de Castilla-La Mancha. 
"Hispania Antiqua Epigraphica»), las recopilaciones provinciales de inscripciones romanas (de gran utilidad), o la nueva versión del CIL. de las Hispaniae (Editio Altera), para detectar la realidad: una fortísima proporción de los textos epigráficos conocidos corresponde a lápidas, estelas o cipos de carácter funerario ${ }^{1}$.

Así pues, salta a la vista la conveniencia, al menos a priori, que los historiadores de la antigüedad tenemos de usar estos documentos para conocer las sociedades de época romana. En este sentido, desde el siglo xix se empezaron a formular las primeras propuestas con un alcance científico, y a lo largo del siglo $x x$ los estudios se han desarrollado en una mayor cantidad e intensidad, con interés por extraer de este tipo de documentación la información pertinente.

Una de las ideas más tópicas que se tienen, porque la misma no deja de ser una característica que se detecta con facilidad, es precisamente que estos documentos utilizan fórmulas basadas en estereotipos, tales como la dedicatoria a los Manes, la indicación de la edad, a veces la identidad del dedicante (muchas veces su heredero), o las fórmulas finales con diversas variantes: $H(i c) s$ (itus/a) e(st) $s$ (it) $t$ (ibi) $t$ (erra) I(evis) en su desarrollo completo. Este hecho es cierto, pero también lo es el que aparecen notables diferencias regionales, en ocasiones difíciles de explicar o interpretar.

Los epígrafes funerarios han permitido desarrollar magníficos trabajos de recopilación acerca de grupos sociales, como es el caso de los esclavos y libertos, la aparición de elementos religiosos, como el culto a los Manes o a determinadas divinidades ${ }^{2}$. También las lápidas han permitido estudios detallados acerca de los nombres personales utilizados ${ }^{3}$

1 Estudios sobre el progreso de la epigrafía latina en Hispania aparecen recogidos en el volumen de CASTILLO, C., Vestigia Antiquitatis. Escritos de epigrafía y literatura romanas, Pamplona, 1997. También diversos aspectos interesantes aparecen recogidos en los análisis bibliográficos sobre Hispania antigua y romana en la Revue des Études Anciennes. La nueva edición del Corpvs Inscriptionum Latinarum ha sacado ya a la luz en los últimos años varios fasciculos del volumen II (Hispaniae), bajo la dirección de la Academiae Scientiarvm Berolinensis et Brandenbvrgensis.

2 Trabajos clásicos al respecto fueron, por ejemplo, los de MANGAS, J., Esclavos y libertos en la España romana, Salamanca, 1971, de CRESPO, S. y SAGREDO, L., “Las profesiones en la sociedad de Hispania romana", Hispania Antiqua, 6, 1976, págs. 53 y ss., y de VÁzouEz HoYs, A. M., "La religión romana en Hispania: análisis estadístico", Hispania Antiqva, 7, 1977, págs. 7 y ss..

3 Albertos, M. L., La onomástica personal primitiva de Hispania: Tarraconense y Bética,Salamanca, 1966; ABASCAL, J.M., Los nombres personales en las inscripciones latinas de Hispania, Murcia, 1994 
y, sobre todo, acerca de la onomástica en relación con las familias principales de las comunidades cívicas. Nos hallamos ante una serie de trabajos que tienen ya, a estas alturas, una sólida tradición, cada vez mayor, en la Historia Antigua española.

Al margen de los objetos de estudio anteriores, que nadie pone en discusión, la epigrafía funeraria puede ser utilizada en otras líneas complementarias, pero con alcance y metodología algo diferentes. En concreto, nos referimos sobre todo a la utilización estadística de factores que no se refieren a la onomástica, a la condición social, o a los cultos religiosos. Un epitafio latino, por sí mismo, ofrece generalmente muy escasos datos de interés. Aspecto diferente es el de la utilización de los mismos con un criterio estadístico, con el fin de documentar determinadas tendencias de la sociedad ${ }^{4}$. En este sentido, dos líneas diferentes, y en buena parte contradictorias, se han apuntado acerca de estos documentos:

1. Las lápidas latinas como documentos para el estudio de la demografía histórica. De hecho, son los aspectos demográficos los que han atraído el mayor número de las investigaciones, comenzadas en el siglo XIX con la obra clásica de Béloch, que por lo general han estado muy centradas en los fenómenos de desplazamientos o migraciones. También existe ya a estas alturas una larga tradición de planteamiento y discusión acerca de viabilidad o no de avanzar en otros aspectos demográficos, tales como la natalidad o la mortalidad, y las diferencias al respecto de hombres y mujeres. Se trata de una línea de conocimiento muy bien expresada en la discusión al respecto de los profesores Robert Etienne y Louis Henry, referida a la validez de las edades de defunción recogidas en las lápidas, y a las posibilidades de obtener conclusiones estadísticas sobre la esperanza de vida en época romana ${ }^{5}$.

Baste indicar que en principio existió al respecto bastante optimismo, postura que incluso se ha venido repitiendo con posterioridad en el

4 Al respecto, Pereira Menaut, G., «Problemas de la consideración global de las inscripciones epigráficas latinas», Papeles del Laboratorio de Arqueología de Valencia, 9, 1973, págs. 125-152; VVAA, "Aplications a l'épigraphie des métodes de l'Informatique", Antiquités Africaines, 9, 1975, págs. 7-151.

5 ETIENNE, R., "Démographie et Epigraphie», Atti del Terzo Congresso Internazionale di Epigrafia Greca e Latina, Roma, 1959, págs. 415-424; HENRY, L., “La mortalité d'après les inscriptions funeraires", Population, 12, 1957, págs. 149-152; IDEM, “L'Age du décès d'après les inscriptions funéraires", Population, 14, 1959, págs. 323-329. 
análisis anexo a muchos repertorios epigráficos. No obstante, la visión optimista acerca del uso de esta información quedó muy corregida y recortada con los principales estudios sobre la cuestión, realizados en los años setenta. En la discusión recogida en los mismos quedaba bastante claro que las lápidas son documentos que tienen unas grandes potencialidades informativas pero que, en todo caso, los investigadores debemos tener mucho cuidado a la hora de trabajar con los datos referidos a la edad de fallecimiento expresada en los mismos ${ }^{6}$. Diversos factores hacen que esas edades estén alteradas en relación a la estructura demográfica real de la sociedad romana.

2. Otra línea de trabajo apunta al carácter más discutible de los epitafios como fuentes demográficas, debido a las peculiaridades del material recogido en los mismos, pero que serían mucho más potentes como documentos en el terreno de lo sociológico. De hecho, los epígrafes funerarios latinos muestran sesgos estadísticos que parecen señalar la inadecuación de un carácter representativo para el estudio de la demografía. Datos básicos al respecto los encontramos, por ejemplo, en la ausencia casi absoluta de la mortalidad infantil (aproximadamente uno de cada cuatro nacidos vivos hasta el siglo xVIII en Europa occidental), la aplastante proporción de las poblaciones urbanas sobre las rurales, el peso desproporcionadamente mayor de los sectores sociales altos o medios (lo que no implica que no aparezcan, aunque en menor proporción, los sectores más bajos y mayoritarios) ${ }^{\text {? }}$.

Partimos de una consideración inicial, que debe motivar la prudencia en los estudios de representatividad: epitafio funerario no se ponía en época romana a todo el mundo. Primero, por una cuestión económica: parece evidente que suponía un gasto que podía ser elevado para muchas (demasiadas) economías. Y segundo, porque el fenómeno de la lápida en la tumba aparece como una posibilidad opcional, pero no como una costumbre que fuera considerada casi imprescindible. Las excavaciones arqueológicas que se realizan en distintas regiones de Hispaniae, señalan como en las necrópolis romanas, de los

\footnotetext{
- Salmon, P., Population et dépopulation dans l'Empire romain, Bruselas, 1974; GaRcia Merino, C., Análisis sobre el estudio de la demografía de la Antigüedad y un nuevo método para la época romana, Valladolid, 1974, como estudios fundamentales sobre la cuestión.

Muy recientemente, CREspo, S. y Alonso, A., "La precisión en la edad de la muerte en la epigrafia funeraria de Hispania", Homenaje a José María Blázquez, IV, Madrid, 1999, págs. 95-119.
} 
siglos I al III, la mayor parte de las tumbas carecían de epitafio. Factores todos ellos que no impiden un estudio demográfico, pero que incluyen muchas dudas al respecto de la representatividad de los epígrafes en relación al conjunto de la sociedad romana. No tener en cuenta este factor puede conducir a conclusiones poco contrastadas con la crítica metodológica.

Por el contrario, los epitafios latinos nos informan con bastante detalle de las principales características de la sociología funeraria de los romanos, incluso de sus grandes variantes locales y regionales. El comportamiento ante la muerte, expresado en unas mayores tendencias señaladas de forma estadística, puede aportar elementos para el conocimiento de la sociedad romana, de un lado, y también de la Historia local silenciada por otras fuentes de información. Es cierto que el método también presenta sus dudas, no sólo referidas a la representatividad de los epígrafes, sino también a la dirección de la interpretación. En efecto, la peculiar sociología funeraria pudo tener un origen tanto en las tendencias de una determinada sociedad, como en las actitudes y aprendizajes de un determinado taller lapicista.

¿Fuentes demográficas o fuentes sociológicas? Naturalmente, la discusión a este respecto puede ser interminable y fundamentada en bases poco sólidas, con argumentos no definitivos. Por esta razón, es necesario profundizar con el estudio detallado de casos concretos, tanto de ciudades antiguas o comarcas, o mejor de territorios algo más amplios, como las provincias (en la división actual). La primera opción queda reducida a unos casos poco numerosos, dada la necesidad de que existan grandes colecciones epigráficas procedentes de una ciudad antigua. Dicha condición, imprescindible en un estudio estadístico (que la muestra sea lo suficientemente amplia), tan sólo se produce en muy contadas ciudades de las Hispaniae. El estudio de conjuntos provinciales (actuales) tiene la virtud de salvar muchas veces el problema del número, pero incluye una gran dispersión geográfica, con unificación que muchas veces es artificial para la antigüedad (distintos conventa jurídicos o provincias).

Probablemente, la cuestión de la proporción de las mujeres en los epitafios romanos resulte muy clarificadora de las cuestiones que planteamos. En este factor encontramos una plena coincidencia en todos los estudios realizados, tanto en el tiempo, como en una enorme diversidad de espacios: las muestras estadísticas de epitafios romanos siempre son más numerosas en los hombres que en las mujeres. Al 
constatar este hecho, Carmen García Merino planteaba en 1974 una serie de preguntas razonables desde el punto de vista del conocimiento científico: “¿Responde esa escasez a una inferioridad numérica real del sexo femenino a partir de los 20 años sobre todo.....? ¿ Acaso a una preferencia por parte de los padres hacia los hijos más que hacia las hijas, tendencia nada nueva en el mundo clásico? ¿Es que la costumbre generalizada de rememorar con más frecuencia a los hombres muestra que los epitafios dedicados a mujeres revisten cierto carácter excepcional?» 8 .

Al final de cuentas, seguimos con la misma pregunta anterior: ¿demografía o sociología funeraria?. No han faltado precisamente las respuestas que han apuntado a favor de la primera interpretación. Por poner un ejemplo, Russell señaló como explicación el que muchas mujeres sobrevivirian a sus maridos, falleciendo después, por lo que ya no recibirían el honor de un epitafio ${ }^{9}$. El hecho sería no tanto el producto de una mayor esperanza de vida femenina, como de la profunda desigualdad del matrimonio romano, al que la mujer llegaba entre 6 y 12 años más joven que su esposo ${ }^{10}$. Es posible también que, como se ha apuntado en ocasiones, existieran muchos más hombres que mujeres, debido a la práctica del infanticidio, y a la exposición de niños, que afectaría mucho más a las hembras que a los varones ${ }^{11}$. No obstante, el profesor Moretti apuntó en otra línea muy diferente, al interpretar que no existía tamaña desproporción demográfica, sino que sería el producto de la falta de consideración social de la mujer ${ }^{12}$.

Así pues, en principio tenemos tres explicaciones para la fuerte y extendida desproporción entre los sexos en los epitafios. Los planteamientos son los siguientes:

$\left.1^{\circ}\right)$ Mayor supervivencia de las mujeres en el matrimonio, lo que conllevaría a la existencia de más viudas que viudos (como en la

Garcia Merino, C., pág. 35.

9 RusselL, J. C., "Late Ancient and Medieval Population", Transactions of the American Philosophical Society, 48, 1958, págs. 1-152.

10 Hecho magníficamente destacado y analizado en sus efectos por GRMEK, M. D., Les maladies a l'aube de la civilisation ooccidentale, Paris, 1993.

1 Salmon, P., pág. 81, que discute no el hecho de que se produjeran sino el que pudieran tener tan fuerte incidencia en la población.

12 MORETTI, L., "Statistica demográfica ed epigrafia: durata media della vita in Roma imperiale», Epigraphica, 21, 1959, págs. 60-78. 
actualidad). En el caso de la época romana, no supondría necesariamente una mayor "esperanza de vida" en las mujeres debido a la mencionada desigualdad en el matrimonio (hipótesis de Russell).

$2^{\circ}$ ) Existencia real de una fuerte desproporción numérica a favor de los hombres en la edad adulta, que se produciría por una mayor supervivencia. La misma vendría determinada por el mayor interés de los padres en los hijos varones, y por un sufrimiento prematuro de las mujeres en la infancia y adolescencia. Es la hipótesis sostenida por Degrassi.

$\left.3^{\circ}\right)$ Inexistencia de desproporción demográfica significativa, sino de una muy fuerte diferenciación social de las mujeres, que en menor proporción merecerían el homenaje y gasto del epitafio. Sería, por tanto, un fenómeno no demográfico sino de sociología funeraria: mayor recuerdo hacia los hombres fallecidos. Como señaló Pierre Salmon, "on ne peut donc se fonder sur les inscriptions funéraires pour établir la proportion des deux sexes" ${ }^{13}$.

Para que se cumpliera el primer caso deberíamos encontrarnos con el hecho de que una buena proporción de los epígrafes funerarios fuera dedicado por las mujeres a sus maridos fallecidos. En ocasiones, dependiendo en su proporción de las zonas, en los epitafios latinos aparece la identidad de quien dedica la lápida, su relación con el fallecido. En las colecciones epigráficas de las Hispaniae tenemos abundantes datos al respecto, pero por lo general no han sido apenas objeto de atención. Por ejemplo, podemos destacar los casos que se refieren a territorios del ámbito de algunas actuales provincias del interior de España, y cuyo análisis consideramos significativo.

En el ámbito territorial de la actual provincia de Madrid, en la recopilación epigráfica de Knapp, tenemos una cierta (aunque minoritaria) expresión de la identidad de los dedicantes de los epitafios. Los casos más numerosos responden a la siguiente casuística: los epitafios puestos por el padre del/la fallecido, los puestos por el hijo, o por la mujer en recuerdo de su esposo; en segunda posición encontramos los epitafios puestos por la madre del/la fallecido/a; en tercer lugar, los epitafios puestos por el marido o por la hija del/la fallecido/a ${ }^{14}$.

13 Salmon, P., pág. 81.

14 KNAPP, R. C., Latin Inscriptions from Central Spain, Berkeley-Los Ángeles, 1992, pág. 389. 
Es cierto que hay más epitafios puestos por las esposas a sus maridos pero podemos preguntarnos hasta qué punto ello puede explicar la desproporción. Es muy dudoso si tenemos en cuenta, además, que el mayor número de lápidas (en total 7 ) vienen representadas por las dedicatorias de padres a sus hijos, seguidas por las dedicadas entre esposos, y las dedicadas por los hijos a sus padres (6 en cada caso). Las relaciones paterno-filiales priman notablemente sobre las conyugales, con lo que resulta muy difícil que la desproporción del bajo número de mujeres venga motivada por la supervivencia de las esposas.

Más claro todavía es al respecto el ejemplo de la serie epigráfica procedente del ámbito de la actual provincia de Segovia, a partir de la recopilación ya mencionada de Knapp. Encontramos en este caso una proporción aceptable de la mención del dedicante de la lápida. El que lo hace en un mayor número de ocasiones es el hijo del fallecido/a, seguido del padre del fallecido, y a bastante distancia ya, la madre y el marido. Ocupando posiciones minoritarias, los epitafios dedicados por la esposa o por la hija. Los resultados de la colección epigráfica de la provincia de Segovia no dejan lugar a las dudas con este caso concreto: predominan los epitafios dedicados por los padres a los hijos y por los hijos a los padres, 8 en cada caso, y muy lejos aparecen después las lápidas dedicadas entre esposos, solamente 5 . Así pues, el predominio de las relaciones paterno-filiales sobre las conyugales es aplastante.

El estudio de otros conjuntos epigráficos no modifica, en absoluto, este tipo de tendencias estadísticas. En la epigrafía de la provincia de Guadalajara, no muy numerosa precisamente, la identificación del dedicante se produce en muy pocas ocasiones: en ellas, el número de las menciones del marido (o contubernal masculino en su caso) es igual a las de la esposa, o de las del hijo a sus padres ${ }^{15}$. Tampoco nos parece muy significativa la estadística de la epigrafía latina de Albacete que, siendo escasa, también menciona raras veces la identidad del dedicante.

Mucho mayor valor presenta una muestra epigráfica concreta, la de Segóbriga, en la actual provincia de Cuenca. La sociología funeraria de

\footnotetext{
15 Abascal, J. M., «Epigrafía romana de la provincia de Guadalajara», Wad-al-Hayara, 10, 1983, págs. 49-115, al que deben unirse algunos epitafios publicados con posterioridad. Al respecto podrá verse, GozAlBES, E., “Aspectos de la sociología funeraria en la Celtiberia meridional», en prensa (en la misma revista mencionada más arriba).
} 
esta ciudad es peculiar en muchos sentidos, y dentro de sus características encierra la fuerte cantidad de ocasiones en las que se menciona la identidad del dedicante del epitafio ${ }^{16}$. Este hecho señala que, aún siendo un caso local y poder constituir una variante, tiene un carácter más significativo que otras colecciones epigráficas. En Segóbriga, el número mayor corresponde a las lápidas puestas por los hijos a sus padres ( $32 ' 56 \%$ de las que reflejan el dato), seguidas de las puestas por los padres a sus hijos (30'23\%), por los epitafios dedicados (de forma equilibrada entre los dos componentes) entre esposos (23'25\%), y muy alejados ya, las lápidas dedicadas entre hermanos (11'62\%). En este caso, todavía resulta más aplastante el predominio de las relaciones paterno-filiales sobre las conyugales en los epitafios: el $62 ' 79 \%$ en el primer caso, frente a sólo el $23{ }^{\prime} 25 \%$ del segundo.

En suma, la primera de las explicaciones apuntada, a la luz de los datos ahora conocidos, parece descartarse. Los casos en los que se menciona la identidad de quien dedica la lápida no parecen señalar ninguna supervivencia mayor de las mujeres con respecto a sus maridos. Naturalmente, estos datos deducidos de las colecciones epigráficas del centro peninsular pueden tener su contraste con los de otras zonas, para ofrecer un panorama mucho más definitivo ${ }^{17}$. Los casos estudiados, y la puesta en común de otros conjuntos regionales, no apuntan en esa dirección.

La duda queda, pues, planteada entre la existencia de una real desproporción de hombres y mujeres, con un número mucho menor de las segundas, o bien en el reflejo del papel subordinado de las mujeres en la sociedad romana. Desde el punto de vista demográfico resulta difícil mantener la existencia de un desequilibrio muy fuerte entre los sexos. No cabe duda de que el número de hombres fue mucho mayor

16 El repertorio fundamental de la epigrafía de Segóbriga es el de ALMAGRo BASCH, Martín, Segóbriga II. Inscripciones ibéricas, latinas paganas y latinas cristianas, Madrid, 1984. Posteriormente se han publicado algunos nuevos epitafios aunque en pequeña cantidad; REverte, J. M.. “Un recinto funerario en Las Obradas de Gaspar, Segóbriga», Revista Cuenca, 31-32, 1988, págs. 93-103; ABASCAL, J. M. y CEBRIÁN, R., "Inscripciones romanas de Segóbriga (1995-1998)", Sagvntvm, 32, 2000, págs. 199-214.

Por ejemplo, en las series epigráficas de la provincia de Valencia detectamos la existencia de más epitafios dedicadas por las mujeres a sus compañeros fallecidos; por el contrario, en la provincia de Jaén no existe esa desproporción, y ambos casos son interesantes por representar colecciones epigráficas muy numerosas. En estos dos casos, además, existen más epitafios dedicados entre esposos que entre padres e hijos. 
en determinadas zonas concretas, especialmente relacionadas con la presencia del ejército. En el resto, resulta difícil que prácticas como el infanticidio, la peor alimentación o trato en la infancia, o la exposición o venta de las hijas, afectara hasta proporciones tan desmesuradas. Incidencia mucho mayor pudieron tener, sin embargo, las muertes derivadas del primer parto en una muy prematura juventud (las mujeres eran casadas entre los 12 y los 14 años en una fuerte proporción).

En todo caso, es interesante tener en cuenta algunas cifras estadísticas. A este respecto, en demografía se ha establecido la denominada sex ratio, que indica el número de hombres existentes por cada 100 mujeres. La comprobación de nacimientos en la especie humana señala que nace un número ligeramente superior de varones que de hembras: por cada nacimiento de mujer nacen 105 hombres ${ }^{18}$. Así pues, la sex ratio natural en la especie humana es de 105. Naturalmente que las muestras epigráficas latinas tienen problemas de representatividad, pero todo desequilibrio fuerte sobre esta proporción nos estaría indicando la doble dirección: un problema demográfico, o un problema sociológico (a los dos sexos no se le dedicaban epitafios por igual).

La desproporción entre hombres y mujeres constituye una constante en todas las colecciones epigráficas del Imperio romano. El estudio comparativo debe hacerse con los epígrafes funerarios exclusivamente, ya que las inscripciones honorarias o religiosas dan muestras mucho más sesgadas acerca de la preponderancia social masculina. La cifra más reducida en el desequilibrio es la resultante de las Hispaniae romanas. Estudiando un conjunto de casi dos mil epitafios, recogidos en la primera edición del CIL., la sex ratio en la Península Ibérica aparece centrada en 126, lo que parece indicar una propoción relativamente ajustada ${ }^{19}$. Otras cifras del Imperio son bastante más altas: 131 en la ciudad de Roma, 139 en Egipto, 140 en Italia, 140-146 en el Africa Proconsular ${ }^{20}$. En otras provincias, las cifras son todavía

18 En realidad, es producto puro del nacimiento. Después la mortalidad natural es ligeramente superior en los niños que en las niñas, con lo que en la mayor parte de las poblaciones el número de componentes de ambos sexos se equilibra, elemento que tiene validez para la época romana; SALMON, P., pág. 80.

19 SALMON, P., pág. 80. La cifra corresponde a 1111 epitafios masculinos y 885 lápidas de mujeres.

20 En total, 4575 epitafios masculinos y 3490 femeninos en Roma; 1055 masculinos y 754 femeninos en el resto de Italia, 6238 epitafios masculinos y 4459 femeninos en el África Proconsular. 
más desequilibradas, debido a la fuerte proporción de los militares en la muestra epigráfica: así nosotros hemos calculado la cifra de 185 en la Mauritania Tingitana ${ }^{21}$, y también 185 es la cifra media en las provincias de Rhetia, Nórica y Pannonia, hasta 196 se eleva la de Britannia...

En el conjunto de las Hispaniae, la publicación creciente de epitafios permite unos estudios regionales con cifras más precisas. De forma excepcional, aparecen casos en los que se conocen más epitafios de mujeres que de hombres. En todas las ocasiones son colecciones locales, o de una comarca muy restringida, que ofrecen datos que son muy poco significativos desde el punto de vista estadístico. Por el contrario, en el contexto de muestras de territorios algo más extensos, invariablemente, los hombres aparecen algo o mucho más numerosos que las mujeres. Aún y así, las cifras son lo suficientemente diferentes como para que resulte congruente tratar de reflejar unas diferencias regionales muy acusadas.

Por lo general, las cifras más bajas de sex ratio en Hispania las encontramos en las series epigráficas de la provincia romana de la Béti$\mathrm{ca}^{22}$. Hay también, no obstante, algunas diferencias entre unas zonas y otras. En algunos casos, como en la Baeturia, o en zonas del convento astigitano (interior de la actual provincia de Málaga) las cifras se elevan a 130-140, excepciones en el contexto de una provincia con más fuerte presencia de mujeres. Por ejemplo, en la ciudad de Gades (uno de los principales puertos de Hispania), con unos 200 epitafios estudiados, la sex ratio es de 111, por tanto, muy ligeramente superior a la natural ${ }^{23}$.

21 Gozalbes, E., «Notas sobre culto y sociología funeraria romana: el caso del municipio romano de Volúbilis (Mavritania Tingitana)", Religio Deorvm. Actas del Coloquio Internacional de Epigrafia (culto y sociedad en Occidente), Sabadell, 1993, págs. 295-301. Por otra parte, en esta muestra epigráfica, 38 epitafios aparecen expresamente dedicados por los padres a su hijo/a fallecido, 21 dedicados al conyuge fallecido, y sólo 10 por los hijos a sus padres. A mi juicio, encontramos una muy evidente graduación en los sentimientos. Los hombres constituían los $2 / 3$ de los fallecidos que recibieron epitafios, y a su vez, eran hombres el 68 '5\% de los dedicantes.

22 Los datos de la Lusitania parecen indicar una diferencia entre algunos centros urbanos y el medio rural o de poblaciones más pequeñas. El estudio realizado por Szilagy, con epitafios que recogen la edad, reflejó en Emerita y Olisipo un total de 145 hombres y 133 mujeres; GaRcía Merino, C., pág. 22. Ello evidenciaría una «sex ratio" muy baja, de 109. Por el contrario, en DEL REAL, M. C., "Estudio sobre la mortalidad en la Lusitania romana», Caesaraugusta, 4-5, 1954, págs. 200-203, en el conjunto de la provincia señala que las lápidas con edad son 228 de hombres y 147 de mujeres (muestra seleccionada). Ello supone una «sex ratio" de 155.

${ }_{23}$ ARIAS, C. y CASTILLO, A. DEL, «Estudio sobre metodología demográfica. El caso de una ciudad andaluza en época romana", Actas I Congreso de Historia de Andalucía. I. Andalucía en la Antigüedad, Córdoba, 1978, págs. 193-201. 
En el otro extremo de la actual Andalucía, en el conjunto de las provincias de Granada y Almería (cuyas tierras se integraban en la mayor parte en la Bética, pero con partes sustanciales también en la Tarraconenses), con una colección de epitafios inferior a la mitad de la anterior, la sex ratio la hemos calculado en 116 , otra cifra que parece muy baja en relación a otras ${ }^{24}$. En el territorio bético de la provincia de Jaén hemos calculado la sex ratio en 118, otra cifra que nos parece muy baja y coherente con las anteriores ${ }^{25}$. Otras cifras son coherentes, así la cifra de 117 es la que se deduce de la colección epigráfica (unos 400 epitafios) de la provincia de Córdoba ${ }^{26}$. En la otra parte de la provincia de Jaén, zona más meridional del Conventus Carthaginiensis (en torno a Castulo), también la "sex ratio" que hemos calculado es muy baja: 115 .

Así pues, en Hispania tenemos una cifra media baja, centrada en algunos cálculos en 126, y en la Bética y sus aledaños de la Tarraconense meridional, unas cifras más bajas todavía, como hemos visto de forma muy normal entre los 111 y los 118 hombres por cada 100 mujeres. Naturalmente, a priori, la cuestión podría plantearse desde la creencia de que existía una mayor proporción de mujeres que en otros lugares del Imperio. Parece muy dudosa la cuestión, acerca de la cual además tendrían que aportarse explicaciones. Por el contrario, nos parece mucho más probable la interpretación de carácter alternativo: sería una muestra de la mayor consideración social de la mujer, que se expresa en la mayor dedicatoria a ellas de lápidas después de muertas. No podemos olvidar que, con otros indicios diferentes, Maria Henar Gallego ha destacado la mayor consideración social de la mujer en la Hispania antigua ${ }^{27}$. Esta mayor consideración social de la mujer hispana podría

24 Los datos los hemos tabulado a partir de los repertorios de PASTOR, M. y MENDOZA, A., Inscripciones latinas de la provincia de Granada, Granada, 1987 y LÁZARO, R., Inscripciones romanas de Almería, Almeria, 1980.

25 Los datos los hemos tabulado a partir de la recopilación de CONZÁLEZ Román, C. y Mangas, J., Corpus de inscripciones latinas de Andalucía. Jaén, 2 tomos, Sevilla, 1991.

26 Galeano Cuenca, G., Costumbres religiosas y prácticas funerarias romanas. Estudio del mundo rural en la provincia de Córdoba, Córdoba, 1997; IDEM, «Epigrafía romana de la provincia de Córdoba", Florentia lliberritana, 8, 1997, págs. 471-534, recoge los epígrates que hemos tabulado.

27 Gallego, M. H., Femina dignísima. Mujer y sociedad en la Hispania antigua, Valiadolid, 1991 ; IDEM, «La consideración en torno a la mujer y su proyección en la sociedad de la Hispania antigua», Hispania Antiqva, 16, 1992, págs. 345-362; IDEM, "Laudationes impensa funeris, locus sepulturae: la mujer y los honores funerarios en Hispania», Hispania Antiqva, 18, 1994, págs. 267-275. 
encontrar en los sentimientos, en el recuerdo y homenaje después de muerta, una expresión bastante significativa.

De ser cierta esta interpretación que apuntamos, conjuntos epigráficos bastante numerosos podrían aportar indicios acerca de la diferente percepción social de la mujer en ámbitos regionales. Sociología funeraria de mentalidad más urbana, o de mentalidad más rural, de zonas interiores o de zonas más costeras, de unas tradiciones indígenas o de otras. Las explicaciones podrían ser diversas, pero se trata sobre todo de detectar si hay diferencias regionales. Hemos visto cifras bastante coincidentes en la zona meridional hispana, tanto en territorios específicamente costeros (Gades), como en áreas del interior incluso montañosas (Jaén, Granada).

Nuestro estudio va a centrarse en las zonas del interior peninsular ${ }^{28}$. El primer indicio al respecto lo presentaba un estudio de Alberto Balil, hace ya casi medio siglo, dedicado a la «duración media de la vida en la Carpetania romana". Utilizaba para ello los epígrafes recogidos en la anticuada obra de Fidel Fuidio acerca de esa región. Destacaban por número de epígrafes, Talavera de la Reina, Toledo y Alcalá de Henares. El autor se preocupaba específicamente de la cuestión de las edades de defunción, en las que encontraba una variante sobre lo más frecuente, como era que las fechas de las mujeres eran mucho más altas que las de los hombres. Centrado en la cuestión no destacó un dato que parece más significativo: la muestra estudiada tenía 34 lápidas de hombres y sólo 15 de mujeres ${ }^{29}$. La "sex ratio" era desproporcionada a todas las luces, 220, y no se incidía en la misma. Con ello, a mi juicio, no se despejaba para nada una más que evidente duda: la alta edad de fallecimiento de las mujeres, 48 años, podía venir provocada por una selección de las fallecidas, con mayor dedicatoria de lápidas a partir de unas determinadas edades ${ }^{30}$. En este sentido, la cifra significativa no vendría

28 Dejamos de lado en este estudio los datos referidos a la Meseta Norte. En todo caso, respecto a las tendencias, la tabulación que hemos realizado de la recopilación hecha en su día por Carmen García Merino refleja una "sex ratio" de 170 en el conjunto del extenso Conventus Cluniensis, con una muestra epigráfica de 410 individuos; García Merino, C., Población y poblamiento en Hispania romana: el Conventus Cluniensis, Valladolid, 1975.

29 BALIL, A., "La duración media de la vida en la Carpetania romana", Revista de Archivos, Bibliotecas y Museos, 61, 1955, págs. 287-292.

30 En las muestras epigráficas romanas, las mayores proporciones de defunción siempre se concentran entre los 20 y los 30 años. En las edades recogidas por Balil este hecho se produce en los hombres de forma muy moderada, pero no en las mujeres. 
marcada por la edad sino por la desproporción de las mujeres, indicadora de una menor dedicatoria de epitafios.

La cifra de la «sex ratio» parece confirmarse con otros hallazgos posteriores. En efecto, hemos recogido un total de 23 nuevos personajes en los epitafios latinos que se han descubierto en la provincia de Toledo en fechas recientes ${ }^{31}$. De ellos hay 16 hombres y solamente 7 mujeres. Ello supone una "sex ratio" de 229 , que viene a coincidir con la deducida con anterioridad. Este hecho parece indicar que las nuevas aportaciones de inscripciones funerarias pueden trastocar el cuadro de las edades, y las conclusiones al respecto, pero no el de la escasísima proporción de mujeres ${ }^{32}$.

Lo mismo podemos observar en la otra parte de la Carpetania, en el ámbito de la actual provincia de Madrid. Desde la publicación de los datos de Fuidio, retomados por Alberto Balil, también se han realizado numerosísimos hallazgos. En la ya mencionada obra de Knapp aparecen recogidas las inscripciones antes conocidas y las aparecidas con posterioridad. Aparentemente se deduce un fenómeno de una proporción que es ligeramente mayor de recogida de la edad de defunción en los hombres que en las mujeres. De esta forma, respecto a fallecidos con edad expresada, Knapp recoge unas cifras que expresarían una «sex ratio» de 300 , cifra desmesurada ${ }^{33}$. Si tenemos en cuenta el conjunto de los epitafios, no sólo los que recogen la edad, hemos calculado la «sex ratio en 268. ¿Alguien puede postular seriamente que había 2'7 hombres por cada mujer en esta zona de la Carpetania?

Dos muestras epigráficas provinciales, en la parte oriental del interior de las Hispaniae, presentan datos poco fiables al tratarse de colecciones

31 ALföLdY, G., «Epigraphica Hispanica X. Inschriften von Unfreien aus Toledo und Umgebung", Zeitschrift für Papyrologie und Epigraphik, 67, 1987, págs. 249-259; MANGAS, J., CARBoles, J. y Rodriguez, S., "Nuevas inscripciones de la provincia de Toledo. I", Hispania Antiqva, 16, 1992, págs. 239-272; IDEM, "Nuevas inscripciones latinas de la provincia de Toledo II", Memorias de Historia Antigua, 17, 1996, págs. 239-254.

32 En estos nuevos epígrafes, la edad de mortalidad media de los hombres aparece fijada en los 40 años, y la de las mujeres en los $35^{\prime} 5$ años. Son datos fuertemente contradictorios con los antes conocidos, y que invierten la tendencia de mortalidad observada en aquellos.

${ }_{33}$ KNAPP, R. C., pág. 386. Sobre todo, en la zona de Complvtvm las diferencias son enormes, con muy pocos epitafios femeninos, pero la desproporción es elevada en toda la provincia. Otro dato peculiar de la epigrafia funeraria madrileña es que, al contrario que en la gran mayoria de las colecciones provinciales, la edad media de defunción de las mujeres es muchísimo más elevada que en los hombres, con una diferencia de ocho años. 
epigráficas con un número muy reducido. En todo caso, debemos recogerlas como muestra de que las tendencias estadísticas no varían. En la provincia de Albacete, sobre un total de 34 personas fallecidas con sexo identificado, un total de 24 son hombres, y tan sólo hay 10 mujeres ${ }^{34}$. Ello supone una "sex ratio" de 240 , que encaja a la perfección con las cifras de las provincias de Toledo y de Madrid. Menos significativa es la cifra resultante de la epigrafía latina de Teruel, con apenas 14 hombres y 8 mujeres ${ }^{35}$. La cifra de "sex ratio" de 175 es, sin duda, poco significativa, pero se inserta también en la misma tendencia que detectamos en todo el interior peninsular.

Nos interesa especialmente tener en cuenta las cifras derivadas de colecciones epigráficas de la Celtiberia meridional, es decir, las actuales provincias de Guadalajara y Cuenca. Vistos los datos anteriores, con una "sex ratio" que prácticamente duplica la de la Hispania meridional, estos datos podrían confirmar o rectificar las tendencias. En primer lugar, la provincia de Guadalajara no tiene una recopilación epigráfica única, puesto que el estudio de Juán Manuel Abascal ha sido completado después con la aparición de nuevos epígrafes. Pues bien, con un total de fallecidos con lápida que no llega al medio centenar, la "Sex ratio" vuelve a mostrarse particularmente elevada: 218 es la cifra que hemos calculado en la provincia de Guadalajara. De nuevo, los datos nos plantean la misma interrogante: ¿acaso puede pensarse que en la zona de Albacete había 2'4 hombres por cada mujer, y en la de Guadalajara 2'2 hombres por cada mujer?

En la actual provincia de Cuenca tenemos otros indicios muy significativos. Es cierto que en la misma podemos detectar diferencias muy sensibles en los datos de la epigrafía de Valeria y de Segóbriga. Este fenómeno no es nada extraño, sino que es resultante de las sensibles divergencias en todos los conceptos, tanto en los formales de los epitafios, como en el texto de los mismos, como en la sociología funeraria que se deduce. En todo caso, siguiendo el análisis provincial que venimos realizando, en el ámbito conquense encontramos unos 180 personajes documentados en inscripciones funerarias, los que representa una cifra muy superior a la de las restantes provincias castellano-manchegas.

34 Datos que hemos tabulado a partir de ABASCAL, J. M., Inscripciones romanas de la provincia de Albacete, Albacete, 1990, completado con ABAScAL, J. M. y SANZ, R., “Novedades de epigrafia romana en la provincia de Albacete", Al-Basit, 33, 1993, págs. 13-35.

35 Datos tabulados a partir de Navarro, M., La epigrafía romana de Teruel, Teruel, 1994. 
En total la "sex ratio" que se deduce de esta muestra epigráfica actualmente conocida es de 162. Las diferencias quedan bien marcadas, por cuanto en Valeria ${ }^{36}$ la cifra no llega siquiera a 140. Respecto a las inscripciones con mención de la edad del fallecido, la "sex ratio" es muy similar, 157, lo que parece señalar la inexistencia de diferencias entre hombres y mujeres en la mención de la edad. En la provincia conquense encontramos el reflejo más bajo de proporción de hombres por cada cien mujeres en relación con las provincias del interior peninsular. Coincide además con la provincia que tiene una colección epigráfica más abultada.

\section{CONCLUSIONES}

El prolijo análisis de cifras estadísticas que hemos realizado, a nuestro juicio, aporta algunos indicios para un mejor conocimiento de la sociedad hispano-romana. En el debate sobre el carácter documental de los epitafios latinos la balanza parece inclinarse a favor de su consideración como elementos sociológicos mucho más que demográficos. Es cierto que son muchísimos las inscripciones funerarias conservadas, pero todos los datos señalan que la fijación de una inscripción, con el nombre del fallecido y otros datos, no fue un fenómeno mayoritario: la mayor parte de las tumbas de época romana carecían de inscripción.

En demasiadas ocasiones se ha apuntado el carácter tópico de los epígrafes funerarios latinos. Esta observación, sin duda apropiada en sentido general, ha ocasionado unas limitaciones en los estudios, de tal forma que unas diferencias que son muy nítidas han sido poco destacadas. No nos referimos a este respecto en los aspectos formales, muy bien señalados por Juan Manuel Abascal, que ha mostrado la existencia de estilos diferentes en los talleres lapicistas, centrando su estudio en detalle en las muestras epigráficas de Castilla-La Mancha y Madrid ${ }^{37}$.

Ejemplo al respecto es la utilización de la invocación inicial a los dioses Manes, puesto que en regiones de la Tarraconense aparece la

36 La muestra epigráfica de Valeria está sustancialmente recogida en Rodríguez COLMenero, A., "Cuenca romana. Contribución al estudio epigráfico", Lvcentvm, 1, 1983, págs. 203-237 y 2, 1984, págs. 319-330.

${ }^{37}$ ABASCAL, J. M., "Aproximación formal a la epigrafía romana de la Meseta meridional», Complvtvm. Roma en el interior de la Peninsula lbérica, Alcalá de Henares, 1998, págs. 129-137. 
misma como $D M$, mientras en la Bética y la Lusitania predomina el añadido de "sagrados", es decir, DMS. La fórmula final de $S$ (it) t(ibi) t(erra) I(evis), ciertamente tópica en Hispania, tan sólo aparece muy extendida además en África y en la ciudad de Roma, siendo más excepcional en el resto ${ }^{38}$.

La estadística se ha aplicado las más de las ocasiones a los epitafios latinos tratando de obtener cifras de mortalidad media, a grandes rasgos de "esperanza de vida". Sin embargo, raramente se ha prestado atención al problema que presenta un mayor valor estadístico, por estar generalizado en las colecciones epigráficas: la diferente repartición de los sexos, con una presencia mayor de hombres que de mujeres. A este respecto se pueden aportar explicaciones de carácter demográfico o sociológico. Es probable que en la sociedad romana existieran más hombres que mujeres, producto de la mayor valoración de los primeros, con un mayor cuidado y atención hacia los niños, y un mayor volumen de exposición de las niñas ${ }^{39}$. Pero la actitud de los padres habría sido de una brutalidad generalizada, para poder producir un desequilibrio tan acusado y constante como el que se manifiesta. Sin pruebas es difícil imaginar no la existencia de casos individuales sino de fenómenos generalizados.

El análisis que hemos realizado ha aportado algunas cifras estadísticas que se refieren a las colecciones epigráficas completas actualmente conocidas. En todas ellas se confirma una tasa de masculinidad particularmente elevada. En algunos casos, de ser ciertas las deducciones demográficas, nos encontraríamos con más de dos hombres por cada mujer. A nuestro juicio, este hecho es totalmente inaceptable en toda sociedad histórica realmente existente, con un cierto potencial como la romana. Parece mucho más lógico deducir otra conclusión: el epitafio era opcional, un gasto suplementario en todo enterramiento, y el mismo se acometía en proporciones mucho mayores cuando el fallecido era hombre que cuando era mujer.

Desde este punto de vista, la proporción de hombres y mujeres reflejados en las inscripciones sería un indicio no demográfico sino de

38 LATTIMORE, R., Themes in Greek and Latin Epitaphs, Urbana, 1962, págs. 65 y ss. Vid. el estudio inicial de VIVES, J., "Características regionales de los formularios epigráficos romanos», Actas del Primer Congreso Español de Estudios Clásicos, Madrid, 1958, págs. 485-492.

39 Vid. algunos datos que apuntan a esta tesis en LASSÉre, J. M., Vbique Popvlvs. Peuplement et mouvements de population dans l'Afrique romaine, Paris, 1977, págs. 508-509. 
carácter sociológico, reflejo de la conformación de la sociedad romana. Una mayor presencia de la mujer podría indicar su mayor valoración social en un territorio concreto. Es natural que esa mayor valoración podía venir determinada por elementos bien diferentes pero complementarios, como las normas y costumbres (civilizadas), las tradiciones culturales del medio local (herencia de pueblos indígenas con mayor o menor protagonismo de la mujer), o el nivel de desarrollo económico (que favorecía en mayor medida los gastos funerarios por parte de los supervivientes). En todo caso, unas diferencias que parecen indicar tendencias bastante claras en la sociedad hispano-romana.

De hecho, la epigrafía funeraria de las Hispaniae, con los datos de la primera edición del CIL, es la que muestra mayor presencia de mujeres de todo el Imperio romano. Este hecho parece corroborar los indicios, señalados con otros testimonios, acerca del papel social relativamente elevado de la condición femenina en la España romana. Es muy significativo que la proporción de mujeres, en una media de diversas provincias, de territorios muy diversos, de ciudades y ambientes rurales, de costa, de valle y de zonas de media montaña, resulte en término medio más elevada que la documentada en la ciudad capital del Imperio (126 hombres por cada cien mujeres en las Hispanias, y 131 en la ciudad de Roma).

Pese a todo, la cifra hispana precisa de una cierta discusión. Debemos tener en cuenta la preponderancia numérica, antes y ahora, de las colecciones epigráficas de unas regiones bien determinadas, coincidentes con la Bética y con la Tarraconense marítima. Es cierto que las mismas tenían, al tiempo, una densidad demográfica más elevada, pero no sabemos hasta qué punto en la misma proporción que la aparición de epigrafía. Si hacemos un análisis regional podemos deducir sensibles diferencias precisamente respecto a la proporción de mujeres. En general, parece cumplirse la tendencia regional: a mayor intensidad de epígrafes, mayor proporción de mujeres. Como la epigrafía latina era una producción no única, pero si mayoritariamente urbana, podríamos encontrarnos con un cierto indicio del mayor protagonismo social de las mujeres en los medios urbanos de la Hispania romana. Algún dato parcial referido a la Lusitania parece indicar también este mismo hecho.

Por regiones, es indudable que la mayor proporción de mujeres en los epígrafes funerarios aparece en la Bética. Todas las colecciones provinciales reflejan el mayor número de hombres, por encima de la "sex ratio" normal. Hay casos, ciertamente, en que la proporción es 
bastante más elevada: por ejemplo, en la parte española de la Baeturia hemos calculado una "sex ratio" de 138, de las cifras más elevadas de la zona andaluza ${ }^{40}$. Se trata indudablemente de unos datos excepcionales, puesto que otros conjuntos provinciales analizados rondan la cifra de 116. Son datos significativos porque en los mismos no sólo aparecen ciudades más o menos grandes, sino entidades más rurales, no sólo centros costeros sino también del interior terrestre. Estas mismas cifras aparecen en la parte de Jaén, en torno a la ciudad de Castulo, que pertenecía a la provincia Tarraconense.

Por el contrario, el análisis de los conjuntos provinciales del interior peninsular ofrece unas cifras que son radicalmente diferentes. Como término de referencia para la Meseta Norte, el estudio tradicional del Convento Cluniense recogía una "sex ratio» de 170 (cifra que obtenemos de la recopilación de García Merino). La desproporción entre 116-117 hombres por cada 100 mujeres en la Bética, y 170 hombres por cada 100 mujeres en el Convento Cluniense, es lo suficientemente elevada como para no pasar desapercibida. Pero los ejemplos restantes que hemos analizado inciden mucho más en la cuestión, con cifras que son todavía más extremas.

El análisis de la Carpetania, provincias de Toledo y de Madrid, indica con gran claridad que la proporción de hombres por cada mujer supera ampliamente el doble. No es una excepción, y tendencias muy similares detectamos en las provincias de Segovia y de Albacete. La muestra epigráfica de Teruel es muy modesta, pero parece señalar en la misma dirección. También en la Celtiberia meridional vemos la cifra de 218 en la "sex ratio" de Guadalajara. Mucho más moderada dentro de lo elevado es el índice de la provincia de Cuenca, 162, con una muestra epigráfica que es significativamente superior a la de las otras provincias.

En suma, en torno a 116-117 en la Bética y zonas aledañas, y de 162 a 268 en el interior de la Tarraconense. El tamaño de las muestras, y la persistencia de las tendencias, hace que nos hallemos ante datos estadísticamente significativos. Nos parece difícil de mantener que responda a proporciones reales en la demografía. Por el contrario,

40 Los datos los hemos tabulado a partir del repertorio de CANTO, A. M., Epigrafia romana de la Beturia Céltica, Madrid, 1997. 
mucho más verosímil es explicar las cifras por una paralela discriminación en la relevancia social de la mujer. La misma era particularmente alta en unas zonas de las Hispaniae, las de mayor potencial demográfico, y de mayor peso de la vida urbana. Por el contrario, el papel de la mujer, en la vida y en la muerte, era muy inferior en otras zonas de características diferentes, como eran las del interior peninsular, lo cual tiene su reflejo en la epigrafía funeraria.

Economía, mentalidad y sentimientos producían esa mayor discriminación hacia la mujer en una práctica no imprescindible, como era la de fijar en la tumba un epígrafe, con el recuerdo de su nombre. Pese al silencio, los enterrados sin epitafio también existieron. Obtengamos de ese silencio inevitable, al menos, la constatación de su discriminada existencia. 